\title{
A BROAD IRON LINE IN THE CHANDRA HIGH ENERGY TRANSMISSION GRATING SPECTRUM OF 4U $1705-44$
}

\author{
T. Di Salvo, ${ }^{1}$ R. Iaria, ${ }^{1}$ M. Méndez, ${ }^{2}$ L. Burderi, ${ }^{3}$ G. Lavagetto, ${ }^{1}$ N. R. Robba, ${ }^{1}$ L. Stella, ${ }^{3}$ And M. van der Klis ${ }^{4}$ \\ Received 2005 January 7; accepted 2005 March 9; published 2005 March 16
}

\begin{abstract}
We present the results of a Chandra $30 \mathrm{ks}$ observation of the low-mass X-ray binary and atoll source $4 \mathrm{U}$ 1705-44. Here we concentrate on the study of discrete features in the energy spectrum at energies below $\sim 3 \mathrm{keV}$, as well as on the iron $\mathrm{K} \alpha$ line, using the High Energy Transmission Grating Spectrometer on board the Chandra satellite. Below $3 \mathrm{keV}$, three narrow emission lines are found at 1.47, 2.0, and 2.6 keV. The 1.47 and $2.6 \mathrm{keV}$ lines are probably identified with $\mathrm{Ly} \alpha$ emission from $\mathrm{Mg}$ XII and S XVI, respectively. The identification of the feature at $\sim 2.0 \mathrm{keV}$ is uncertain because of the presence of an instrumental feature at the same energy. The iron $\mathrm{K} \alpha$ line at $\sim 6.5 \mathrm{keV}$ is found to be intrinsically broad (FWHM $\sim 1.2 \mathrm{keV}$ ); its width can be explained by reflection from a cold accretion disk extending down to $\sim 15 \mathrm{~km}$ from the neutron star center or by Compton broadening in the external parts of a hot $(\sim 2 \mathrm{keV})$ Comptonizing corona. We finally report here precise X-ray coordinates of the source.
\end{abstract}

Subject headings: accretion, accretion disks — stars: individual (4U 1705-44) — stars: neutron — $\mathrm{X}$-rays: binaries - X-rays: general $-\mathrm{X}$-rays: stars

Online material: color figures

\section{INTRODUCTION}

Broad emission lines (FWHM up to $\sim 1 \mathrm{keV}$ ) at energies in the range 6.4-6.7 keV are often observed in the spectra of lowmass X-ray binaries, both in systems containing black hole candidates (see, e.g., Miller et al. 2002a, 2002b) and in systems hosting an old accreting neutron star (see, e.g., Barret 2001 for a review). These lines are identified with the $\mathrm{K} \alpha$ radiative transitions of iron at different ionization stages. Sometimes an iron absorption edge at energies of $\sim 7-8 \mathrm{keV}$ has been detected. These features are powerful tools to investigate the structure of the accretion flow close to the central source; in particular, important information can be obtained from detailed spectroscopy of the iron $\mathrm{K} \alpha$ emission line and absorption edge, since these are determined by the ionization stage, geometry, and velocity field of the reprocessing plasma.

To explain the large width of these lines, it has been proposed that they originate from emission reprocessed by the accretion disk surface illuminated by the primary Comptonized spectrum (Fabian et al. 1989). In this model, the combination of relativistic Doppler effects arising from the high orbital velocities and gravitational effects due to the strong field in the vicinity of the neutron star smears the reflected features. Therefore, the line will have a characteristically broad profile, the detailed shape of which depends on the inclination and on how deep the accretion disk extends into the neutron star potential (e.g., Fabian et al. 1989; Stella 1990).

An alternative location of the line-emitting region is the inner part of the so-called accretion disk corona (ADC), probably formed by evaporation of the outer layers of the disk illuminated by the emission of the central object (e.g., White \& Holt 1982). In this case, the width of the line is explained by thermal

\footnotetext{
${ }^{1}$ Dipartimento di Scienze Fisiche ed Astronomiche, Università di Palermo, via Archirafi 36, 90123 Palermo, Italy; disalvo@gifco.fisica.unipa.it.

${ }^{2}$ National Institute for Space Research, Sorbonnelaan 2, 3584 CA Utrecht, Netherlands.

${ }^{3}$ Osservatorio Astronomico di Roma, via Frascati 33, 00040 Monteporzio Catone (Rome), Italy.

${ }^{4}$ Astronomical Institute "Anton Pannekoek," University of Amsterdam and Center for High-Energy Astrophysics, Kruislaan 403, NL 1098 SJ Amsterdam, Netherlands.
}

Comptonization of the line photons in the ADC. This produces a genuinely broad Gaussian distribution of line photons, with $\sigma \geq E_{\mathrm{Fe}}\left(k T_{e} / m_{e} c^{2}\right)^{1 / 2}$, where $E_{\mathrm{Fe}}$ is the centroid energy of the iron line and $k T_{e}$ is the electron temperature in the ADC (see Kallman \& White 1989; Brandt \& Matt 1994 for more detailed calculations). This mechanism can explain the width of the iron line for temperatures of the emitting region of few $\mathrm{keV}$.

The presence of several unresolved components, which can eventually be resolved by the high-resolution X-ray instruments on board Chandra and XMM-Newton, can also contribute to broaden the line.

The low-mass X-ray binary $4 \mathrm{U} 1705-44$ is a very interesting source of the atoll class (see Hasinger \& van der Klis 1989) that also shows type I X-ray bursts. As in other similar sources, the energy spectrum of $4 \mathrm{U} 1705-44$ can be described as the sum of a (dominating) Comptonized component, a blackbody, and an emission line at $\sim 6.4 \mathrm{keV}$. We selected this source for a Chandra observation because a broad (1.1 keV FWHM) iron emission line at $6.5 \mathrm{keV}$ has been previously reported (White et al. 1986; Barret \& Olive 2002). One of the goals of our Chandra observation was to study the iron line profile to discriminate among the various models that have been proposed to explain the large line width. The Chandra High Energy Transmission Grating Spectrometer (HETGS) observation demonstrates that the iron line is intrinsically broad $(1.2 \mathrm{keV}$ FWHM); possible broadening mechanisms are discussed.

\section{OBSERVATIONS}

The source $4 \mathrm{U}$ 1705-44 was observed using the HETGS on board Chandra starting on 2001 July 1. Part of the observation, for a total integration time of $24.4 \mathrm{ks}$, was performed in timed graded mode, while a short part of the observation, $\sim 5 \mathrm{ks}$, was performed in continuous clocking mode. The HETGS consists of two types of transmission gratings, the Medium Energy Grating (MEG) and the High Energy Grating (HEG). The HETGS affords high-resolution spectroscopy from 1.2 to $31 \AA(0.4-10 \mathrm{keV})$ with a peak spectral resolution of $\lambda / \Delta \lambda \sim 1000$ at $12 \AA$ for HEG first order. The dispersed spectra were recorded with an array of six charge-coupled devices 
(CCDs) that are part of the Advanced CCD Imaging Spectrometer-S (Garmire et al. 2003). ${ }^{5}$ The current relative accuracy of the overall wavelength calibration is on the order of $0.05 \%$, leading to a worst-case uncertainty of $0.004 \AA$ in the first-order MEG and $0.006 \AA$ in the first-order HEG. We processed the event lists using available software (FTOOLS and CIAO ver. 3.1 packages). We computed aspect-corrected exposure maps for each spectrum, allowing us to correct for effects from the effective area of the CCD spectrometer.

For the part of the observation performed in timed graded mode, the brightness of the source required additional efforts to mitigate "photon pileup" effects. We applied a 400 raw "subarray" (with the first raw =1) during the observation that reduced the CCD frame time to $1.4 \mathrm{~s}$. The zeroth-order image is affected by heavy pileup; the event rate is so high that two or more events are detected in the CCD during the $1.4 \mathrm{~s}$ frame exposure. Pileup distorts the count spectrum because detected events overlap and their deposited charges are collected into single, apparently more energetic, events. Moreover, many events $(\sim 90 \%)$ are lost as the grades of the piled up events overlap those of highly energetic background particles and are thus rejected by the onboard software. We therefore will ignore the zeroth-order events in the subsequent analysis. On the other hand, the grating spectra are not, or only moderately (less than $10 \%$ ), affected by pileup. In our analysis, we utilize the HEG first-order spectrum, which is less affected by pileup (less than $6 \%$ at maximum, less than $3 \%$ below $3 \mathrm{keV}$ and above $6 \mathrm{keV}$ ) with respect to the MEG first-order spectrum.

To determine the zero-point position in the image as precisely as possible, we calculated the mean crossing point of the zerothorder readout trace and the tracks of dispersed HEG and MEG arms. This results in the following source coordinates: R.A. $=17^{\mathrm{h}} 08^{\mathrm{m}} 54.47$, decl. $=-44^{\circ} 06^{\prime} 07^{\prime \prime} .35$ (J2000.0, uncertainty $\left.0^{\prime \prime} .5\right)$. Note that this position is significantly different $(\sim 0$ '.15) from the coordinates previously reported for this source (see Liu et al. 2001 and references therein).

The data collected in continuous clocking mode do not suffer from photon pileup in the first-order dispersed spectra. However, as a result of the short exposure time of this observation, the statistics are quite low and we only used these data to check the results obtained with the timed graded observation. Indeed, the spectra acquired in continuous clocking mode are in good agreement with the HEG spectra in timed graded mode (both in the shape of the continuum emission and in the parameters of the iron line at $6.5 \mathrm{keV}$; see below). We therefore conclude that the effects of pileup in the HEG spectra of 4U 1705-44 acquired in timed graded mode are negligible.

\section{SPECTRAL ANALYSIS}

We selected the first-order spectra from the HEG. Data were extracted from regions around the grating arms; to avoid overlapping between HEG and MEG data, we used a region size of 26 pixels for the HEG along the cross-dispersion direction. The background spectra were computed, as usual, by extracting data above and below the dispersed flux. The contribution from the background is $\sim 0.3 \%$ of the total count rate. We used the standard CIAO tools to create detector response files (see Davis 2001) for the HEG +1 and -1 order (background-subtracted) spectra, which we fit simultaneously using the XSPEC version 11.2 data analysis package (Arnaud 1996). The HEG spectra were rebinned to $0.005 \AA$.
Two large energy gaps at those wavelengths corresponding to the junction between two CCDs are present in the HEG firstorder spectra, at 3-3.4 keV and 5.5-6.5 keV for the +1 and -1 order, respectively. In these intervals, the effective area is much smaller and is known with less accuracy. ${ }^{6}$ Unfortunately, in the interval between 6 and $6.6 \mathrm{keV}$, the $\mathrm{HEG}+1$ and -1 order spectra give slightly different residuals with respect to the same continuum model. In the present work, we therefore exclude the interval mentioned above corresponding to the junction between two CCDs and in which the two orders give different results. The energy ranges used in the following spectral analysis are 1.3-10 keV for the -1 order and $1.3-6 \mathrm{keV}$ and $6.6-10 \mathrm{keV}$ for the +1 order.

We fit the HEG first-order spectra of $4 \mathrm{U} 1705-44$ to a continuum model. The best-fit model consists of the Comptonization model comptt (Titarchuk 1994), modified by absorption from neutral matter, parametrized by the equivalent hydrogen column $N_{\mathrm{H}}$, which gives a $\chi_{\text {red }}^{2}$ (degrees of freedom [dof]) of 1.05 (3265). This model also includes an overabundance of $\mathrm{Si}$ by a factor of $\sim 2$ with respect to solar abundances to fit a highly significant absorption edge at $\sim 1.84 \mathrm{keV}$ (the addition of this parameter reduces the $\chi^{2}$ by $\Delta \chi^{2} \simeq 61$ at the expense of 1 dof). Note that we cannot exclude that this feature may be due to the presence of $\mathrm{Si}$ in the CCDs, and therefore this overabundance is not discussed further. Finally, in all the fits we include an instrumental feature at $2.06 \mathrm{keV}$ (usually present in the HETG spectra of bright sources; see Miller et al. 2002a, 2002b), which is fitted by an inverse edge (with $\tau \sim-0.1)$.

A soft blackbody component has often been reported in the $\mathrm{X}$-ray spectra of this kind of source and has been detected in the spectra of $4 \mathrm{U} 1705-44$ as well. In $4 \mathrm{U} 1705-44$, in spectral states similar to the one found during our Chandra observation, this component has a temperature of $\sim 1.9 \mathrm{keV}$ and can contribute up to $20 \%$ of the total source flux (Barret \& Olive 2002). We therefore added a blackbody component to the comptt continuum model; this component has a temperature of $\sim 1.95 \mathrm{keV}$ and contributes up to $50 \%$ of the total X-ray flux. However, the decrease of the $\chi^{2}$ for the addition of this component ( $\Delta \chi^{2}=5$ for the addition of two parameters) is not significant, and, for sake of simplicity, we preferred not to include this component in our continuum model.

Residuals in units of $\sigma$ with respect to the continuum model described above are shown in Figure 2; several discrete features are still clearly visible in the residuals with respect to this continuum model, at $\sim 1.5,2.0$, and $2.6 \mathrm{keV}$ and, particularly, in the 6-7 keV range, where the $\mathrm{K} \alpha$ iron emission line is expected. From these residuals, it is apparent that the iron line is intrinsically broad and shows a complex profile.

The addition of a broad $(\sigma \sim 0.5 \mathrm{keV})$ Gaussian line centered at $6.5 \mathrm{keV}$ proves necessary, giving $\Delta \chi^{2}=167$ for the addition of three parameters. We also added three narrow emission lines to fit the other low-energy residuals mentioned above. The addition of Gaussian emission lines at $\sim 1.5,2.0$, and $2.6 \mathrm{keV}$ gives a reduction of the $\chi^{2}$ by 27,29 , and 32 units, respectively, for the addition of three parameters. The errors in the normalizations of these features give a detection at about the $3 \sigma$ confidence level. This is not a highly significant detection and needs a confirmation with future observations. However, the fact that the energies of these features are close to the energies of $\mathrm{Ly} \alpha$ transitions of $\mathrm{H}$-like ionization stages of the most abundant ions emitting in the observed range (that are $\mathrm{Mg}$ XII, 


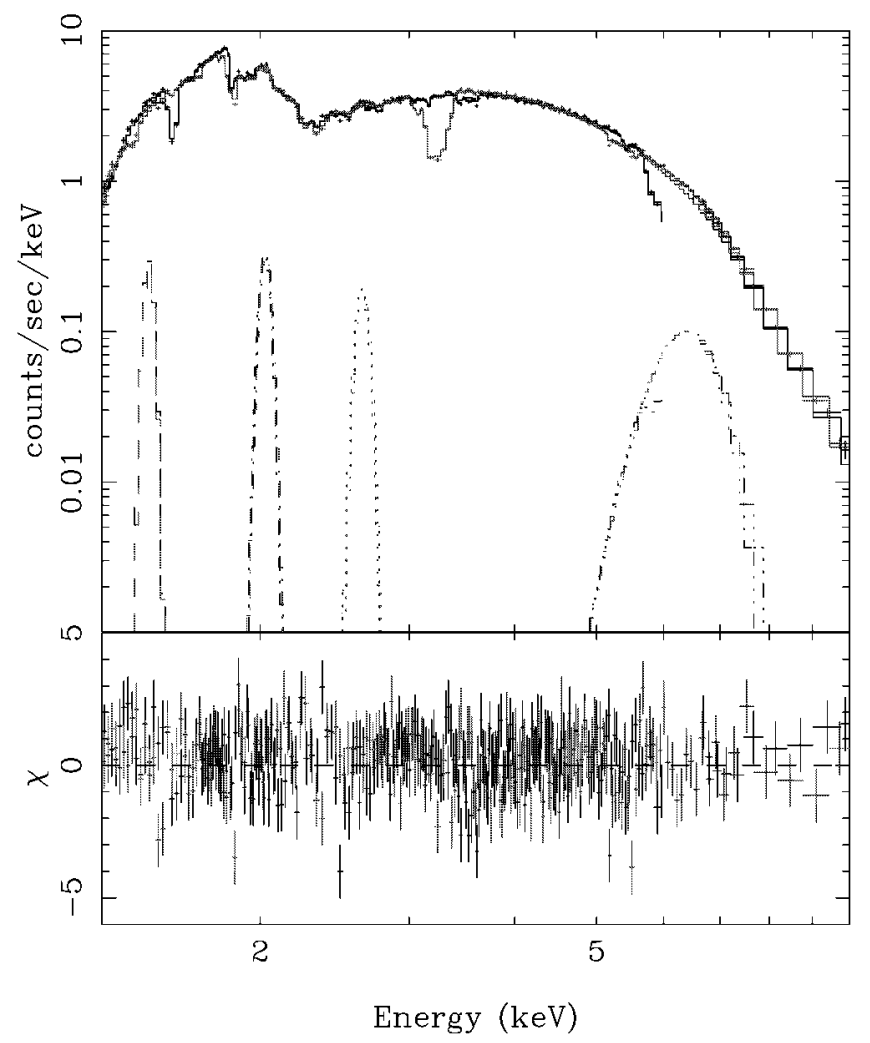

FIG. 1.-Top panel: HEG first-order spectra of 4U 1705-44 together with the best-fit model (see Table 1). The discrete features included in the best-fit model and described by Gaussians are also shown. Note that, for the sake of clarity, we have rebinned the data in the plot with respect to the energy resolution used for the spectral analysis. Bottom panel: Residuals in units of $\sigma$ with respect to the best-fit model. [See the electronic edition of the Journal for a color version of this figure.]

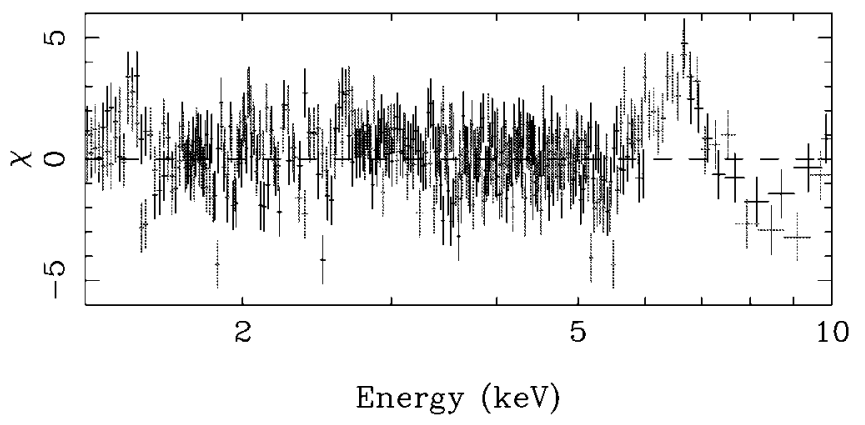

FIG. 2.-Residuals in units of $\sigma$ with respect to the simple Comptonization continuum when the iron line and other low-energy Gaussians are not included in the model. [See the electronic edition of the Journal for a color version of this figure.]

Si XIV, and S XVI, respectively) adds further confidence that these lines may be real. Data and residuals in units of $\sigma$ with respect to this best-fit model are shown in Figure 1. The bestfit model is reported in Table 1 together with the discrete features mentioned above as well as the identification of the line, when possible.

The spectral analysis of the first-order HEG spectra of $4 \mathrm{U}$ 1705-44 from the part ( $\sim 5 \mathrm{ks})$ of the Chandra observation performed in continuous clocking mode confirms the best-fit model found for the timed graded spectra. In particular, the bestfit continuum model is again given by the comptt model, which gives a $\chi^{2}($ dof $)=867$ (1097). The addition of a broad Gaussian emission line at $6.6 \pm 0.1 \mathrm{keV}(\sigma=0.4 \pm 0.1 \mathrm{keV}, \mathrm{EW}=$ $138 \mathrm{eV}$ ) improves the fit, giving a $\chi^{2}($ dof $)=831$ (1094).

The residuals in the K-shell iron line range are quite complex (see Fig. 2), showing a broad feature centered at $\sim 6.5 \mathrm{keV}$. We therefore try to fit this feature with the line profile expected from a thin Keplerian accretion disk. Substituting the Gaussian line with the diskline model (Fabian et al. 1989), we obtain an equivalently good fit, $\chi^{2}$ (dof) $=3164$ (3252), using the diskline model. The line best-fit parameters for the diskline model are given in Table 2.

TABLE 1

Results of the Fitting of THE 4U 1705-44 HEG First-Order Spectra In the $1.3-10 \mathrm{keV}$ ENERGY BAND

\begin{tabular}{|c|c|}
\hline Parameter & Value \\
\hline$N_{\mathrm{H}}\left(10^{22} \mathrm{~cm}^{-2}\right)$ & $1.42 \pm 0.06$ \\
\hline $\mathrm{Si} / \mathrm{Si}_{\odot} \ldots \ldots \ldots$ & $2.0 \pm 0.2$ \\
\hline$k T_{0}(\mathrm{keV})$ & $0.50 \pm 0.02$ \\
\hline$k T_{e}(\mathrm{keV}) \ldots$ & $2.29 \pm 0.09$ \\
\hline & $17.7 \pm 0.7$ \\
\hline Flux $(1.3-10 \mathrm{keV})\left(\mathrm{ergs} \mathrm{cm}^{-2} \mathrm{~s}^{-1}\right)$ & $7.82 \times 10^{-9}$ \\
\hline Final $\chi^{2}$ (dof) $\ldots \ldots \ldots \ldots \ldots \ldots \ldots \ldots \ldots \ldots \ldots$ & $3168(3255)$ \\
\hline$E_{1}(\mathrm{ID}: \mathrm{Mg}$ XII Ly $\alpha)(\mathrm{keV})$ & $1.476 \pm 0.007$ \\
\hline$\sigma_{1}(\mathrm{eV}) \ldots \ldots$ & $17 \pm 6$ \\
\hline$I_{1}\left(10^{-3} \mathrm{~cm}^{-2} \mathrm{~s}^{-1}\right)$ & $2.3 \pm 0.9$ \\
\hline $\mathrm{EW}_{1}(\mathrm{eV}) \ldots \ldots \ldots$ & 4.28 \\
\hline$E_{2}(\mathrm{ID}: \mathrm{Si}$ xIV Ly $\alpha$ ?) $(\mathrm{keV}) \ldots$ & $2.03 \pm 0.01$ \\
\hline$\sigma_{2}(\mathrm{eV}) \ldots \ldots \ldots \ldots \ldots \ldots \ldots$ & $28 \pm 10$ \\
\hline$I_{2}\left(10^{-3} \mathrm{~cm}^{-2} \mathrm{~s}^{-1}\right)$ & $1.9 \pm 0.7$ \\
\hline $\mathrm{EW}_{2}(\mathrm{eV})$ & 4.17 \\
\hline$E_{3}(\mathrm{ID}: \mathrm{S}$ xVI Ly $\alpha)(\mathrm{keV})$ & $2.64 \pm 0.02$ \\
\hline$\sigma_{3}(\mathrm{eV})$ & $40 \pm 14$ \\
\hline$I_{3}\left(10^{-3} \mathrm{~cm}^{-2} \mathrm{~s}^{-1}\right)$ & $2.3 \pm 0.8$ \\
\hline $\mathrm{EW}_{3}(\mathrm{eV}) \ldots$ & 6.32 \\
\hline $\mathrm{E}_{\mathrm{Fe}}(\mathrm{keV}) \ldots$ & $6.54 \pm 0.07$ \\
\hline$\sigma_{\mathrm{Fe}}(\mathrm{keV}) \ldots \ldots$ & $0.51 \pm 0.08$ \\
\hline$I_{\mathrm{Fe}}\left(10^{-2} \mathrm{~cm}^{-2} \mathrm{~s}\right.$ & $1.5 \pm 0.3$ \\
\hline $\mathrm{EW}_{\mathrm{Fe}}(\mathrm{eV})$ & 170 \\
\hline
\end{tabular}

\section{DISCUSSION}

We have analyzed a Chandra 30 ks observation of the Xray burster and atoll source $4 \mathrm{U} 1705-44$. The position of the zeroth-order image of the source provides improved X-ray coordinates for $4 \mathrm{U} 1705-44$ (R.A. $=17^{\mathrm{h}} 08^{\mathrm{m}} 54.47$, decl. $=$ $-44^{\circ} 06^{\prime} 07^{\prime \prime} .35$ ), which significantly differ (by about 0.15 ) from the coordinates previously reported for this source.

We performed a spectral analysis of the HEG first-order spectra of $4 \mathrm{U} 1705-44$. The continuum emission is well fitted by the Comptonization model comptt, with an equivalent hydrogen column of $\sim 1.4 \times 10^{22} \mathrm{~cm}^{-2}$, and an overabundance of $\mathrm{Si}$ by a factor of $\sim 2$ with respect to the solar abundance

TABLE 2

Iron Line Parameters From the diskline MODEL

\begin{tabular}{ll}
\hline \hline \multicolumn{1}{c}{ Parameter } & \multicolumn{1}{c}{ Value } \\
\hline Energy $(\mathrm{keV}) \ldots \ldots \ldots$ & $6.40 \pm 0.04$ \\
$R_{\text {in }}\left(R_{g}\right) \ldots \ldots \ldots \ldots \ldots \ldots$ & $7_{-1}^{+4}(<11)$ \\
$R_{\text {out }}\left(R_{g}\right) \ldots \ldots \ldots \ldots \ldots$ & $410_{-130}^{+230}$ \\
Inclination $(\mathrm{deg}) \ldots \ldots \ldots$ & $59_{-4}^{+25}$ \\
Index $\ldots \ldots \ldots \ldots \ldots \ldots$ & $2.1 \pm 0.2$ \\
$I\left(10^{-2} \mathrm{~cm}^{-2} \mathrm{~s}^{-1}\right) \ldots \ldots$ & $1.8 \pm 0.3$ \\
Final $\chi^{2}($ dof $) \ldots \ldots \ldots$ & $3164(3252)$ \\
\hline
\end{tabular}


(which might be of instrumental origin). The inferred unabsorbed flux of the source in the $0.1-10 \mathrm{keV}$ range is $\sim 1.0 \times$ $10^{-8} \mathrm{ergs} \mathrm{cm}^{-2} \mathrm{~s}^{-1}$, corresponding to a luminosity of $3.3 \times$ $10^{37} \mathrm{ergs} \mathrm{s}^{-1}$ assuming a distance to the source of $7.4 \mathrm{kpc}$ (Haberl \& Titarchuk 1995). The Comptonization continuum is quite soft, with an electron temperature of $k T_{e} \sim 2.3 \mathrm{keV}$ and an optical depth of $\tau \sim 18$ for a spherical geometry.

We have detected a broad emission feature at $6.4-6.5 \mathrm{keV}$, which we interpret as K-shell fluorescent emission of lowly ionized iron. The high-energy resolution of the HEG shows that the line is intrinsically broad (FWHM $\sim 1.2 \mathrm{keV}$ ), in agreement with previous measures (see, e.g., Barret \& Olive 2002). Therefore, the most probable origin of this line is an accretion disk (in this case, the large width of the line would be due to Doppler and relativistic smearing effects) or a hot corona (in this case, the large width of the line would be due to Compton broadening).

In the case the line is produced by reflection in an accretion disk, we estimate that the required inner radius of the disk is $\sim 7 R_{g}$ or $\sim 15 \mathrm{~km}$ for a $1.4 M_{\odot}$ neutron star. Note that the quite small inner radius of the disk inferred from this model is in agreement with the quite soft X-ray spectrum of 4U 1705-44 during the Chandra observation, which would probably place the source in the banana state of its X-ray color-color diagram. In this model, the inclination of the disk with respect to the line of sight is constrained in the range $55^{\circ}-84^{\circ}$. Alternatively, Comptonization in the corona could explain the large width of the line. Detailed calculations give $\sigma_{\mathrm{Fe}}=0.019 E_{\mathrm{Fe}} \tau_{\mathrm{T}}(1+$ $0.78 k T_{e}$ ), where $\tau_{\mathrm{T}}$ is the Thompson optical depth and $k T_{e}$ is in units of $\mathrm{keV}$ (Kallman \& White 1989; see also Brandt \& Matt 1994). Assuming an average electron temperature of $k T_{e}=2.3 \mathrm{keV}$, as derived from the fit of our data to the Comptonization model, we can explain the width of the iron line for a Thomson optical depth of $\tau_{\mathrm{T}} \sim 1.4$. Therefore, it is possible that the line is produced in the outer region of the Comptonizing corona, where the optical depth might be lower (if we assume that the temperature remains constant). This is not unreasonable given that any contribution to the line produced inside the Comptonizing region, where the optical depth can be as high as 10 (see Table 1), would be completely smeared by Comptonization. Therefore, as expected, we only see that part of the line that is produced in the outer Comptonizing region.

Unfortunately, with these data we are not yet able to discriminate between the two possible origins of the iron line and its broadening proposed above. Higher statistics or detection of line variability would be needed for this. Naturally, what would definitively discriminate between the two proposed models is the detection of a double peak in the iron line profile; this would exclude the Comptonization of line photons model and would indicate the relativistic/Doppler effects as the origin of the line broadening. This detection would be possible with higher statistics (large effective areas or long exposures). Hence, more XMM-Newton and Chandra observations (better if simultaneous) would be ideal to this aim. Snapshot observations at different intensity levels and/or spectral states of the source would also be useful to study the variability of the line parameters with the position of the source in the X-ray colorcolor diagram and/or the frequency of the so-called kilohertz quasi-periodic oscillations ( $\mathrm{kHz}$ QPOs). If the source position in the color-color diagram track is indeed determined by the mass accretion rate (see, e.g., Hasinger \& van der Klis 1989) and the frequencies of the $\mathrm{kHz}$ QPOs are related to the Keplerian frequency at the inner edge of the accretion disk (as envisaged by most of the current models; see, e.g., Miller et al. 1998; Stella \& Vietri 1999), then one would expect that the radius of the disk as determined from the line profile (fitted with a diskline model) should change accordingly. These kinds of studies could give other pieces of evidence in favor of one or the other model.

To fit discrete residuals at low energies with respect to the continuum model, we added to the model several Gaussian lines. In Table 1, we report the most significant of these features, together with a possible identification of the line. The lines at 1.5 and $2.6 \mathrm{keV}$ are identified with $\mathrm{Ly} \alpha$ transitions from $\mathrm{H}$-like $\mathrm{Mg}$ at $1.4726 \mathrm{keV}$ (or, less probably, L-shell transitions from highly ionized iron, Fe XXII-Fe XXIV) and S at $2.6227 \mathrm{keV}$, respectively. The identification of the line at $\sim 2.0 \mathrm{keV}$ is more uncertain owing to the presence of a systematic feature at $\sim 2.07 \mathrm{keV}$. Note, however, that this line is quite close to the Ly $\alpha$ transition of Si XIV at $2.0061 \mathrm{keV}$. Finally, we note that there seems to be a correlation between the energy and the width of these lines, with the Gaussian $\sigma$ increasing with the centroid energy of the line. If the line width is due to velocity dispersions, we have calculated that $\Delta E / E_{0}=v / c \sim 2.7 \%-3.6 \%$ (where we have used for $\Delta E$ the FWHM), slightly increasing with the energy of the line, from $\mathrm{Mg}$ XII to $\mathrm{S}$ XVI, as expected if more ionized elements are produced closer to the central X-ray source.

This work was partially supported by the Ministero della Istruzione, della Universitá e della Ricerca.

\section{REFERENCES}

Arnaud, K. A. 1996, in ASP Conf. Ser. 101, Astronomical Data Analysis Software and Systems V, ed. G. H. Jacoby \& J. Barnes (San Francisco: ASP), 17

Barret, D. 2001, Adv. Space Res., 28, 307

Barret, D., \& Olive, J. F. 2002, ApJ, 576, 391

Brandt, W. M., \& Matt, G. 1994, MNRAS, 268, 1051

Davis, J. E. 2001, ApJ, 562, 575

Fabian, A. C., et al. 1989, MNRAS, 238, 729

Garmire, G. P., Bautz, M. W., Ford, P. G., Nousek, J. A., \& Ricker, G. R. 2003, Proc. SPIE, 4851, 28

Haberl, F., \& Titarchuk, L. 1995, A\&A, 299, 414
Hasinger, G., \& van der Klis, M. 1989, A\&A, 225, 79

Kallman, T., \& White, N. E. 1989, ApJ, 341, 955

Liu, Q. Z., van Paradijs, J., \& van den Heuvel, E. P. J. 2001, A\&A, 368, 1021 Miller, J. M., et al. 2002a, ApJ, 578, 348

. 2002b, BAAS, 34, 1206

Miller, M. C., Lamb, F. K., \& Psaltis, D. 1998, ApJ, 508, 791

Stella, L. 1990, Nature, 344, 747

Stella, L., \& Vietri, M. 1999, Phys. Rev. Lett., 82, 17

Titarchuk, L. 1994, ApJ, 434, 570

White, N. E., \& Holt, S. S. 1982, ApJ, 257, 318

White, N. E., et al. 1986, MNRAS, 218, 129 